\title{
Complexities of Next-Generation Sequencing in Solid Tumors: Case Studies
}

\author{
Alexandra O. Sokolova, MD1,2,3; Brian H. Shirts, MD, PhD4; Eric Q. Konnick, MD, MS; Ginger J. Tsai, MS4;
} Bernardo H.L. Goulart, MD1,2; Bruce Montgomery, MD1,2,3; Colin C. Pritchard, MD, PhD4;

Evan Y. Yu, MD ${ }^{1,2}$; and Heather H. Cheng, MD, $\mathrm{PhD}^{1,2}$

\section{ABSTRACT}

With the promise and potential of clinical next-generation sequencing for tumor and germline testing to impact treatment and outcomes of patients with cancer, there are also risks of oversimplification, misinterpretation, and missed opportunities. These issues risk limiting clinical benefit and, at worst, perpetuating false conclusions that could lead to inappropriate treatment selection, avoidable toxicity, and harm to patients. This report presents 5 case studies illustrating challenges and opportunities in clinical nextgeneration sequencing interpretation and clinical application in solid tumor oncologic care. First is a case that dissects the origin of an ATM mutation as originating from a hematopoietic clone rather than the tumor. Second is a case illustrating the potential for tumor sequencing to suggest germline variants associated with a hereditary cancer syndrome. Third are 2 cases showing the potential for variant reclassification of a germline variant of uncertain significance when considered alongside family history and tumor sequencing results. Finally, we describe a case illustrating challenges with using microsatellite instability for predicting tumor response to immune checkpoint inhibitors. The common theme of the case studies is the importance of examining clinical context alongside expert review and interpretation, which together highlight an expanding role for contextual examination and multidisciplinary expert review through molecular tumor boards.

J Natl Compr Canc Netw 2020;18(9):1150-1155 doi: $10.6004 /$ jnccn.2020.7569

${ }^{1}$ Department of Medicine, University of Washington; ${ }^{2}$ Clinical Research Division, Fred Hutchinson Cancer Research Center; ${ }^{3}$ VA Puget Sound Health Care System; and ${ }^{4}$ Department of Laboratory Medicine, University of Washington, Seattle, Washington.

\section{Background}

Over the past decade, advances in next-generation sequencing (NGS) have decreased cost and enabled more widespread use in solid tumor oncology. This has been accompanied by increasing therapeutic options based on tumor genomic profiling, such as ROS1 mutations in lung cancer ${ }^{1}$ and BRAF mutations in melanoma. ${ }^{2}$ In addition, the clinical applications of germline sequencing in solid tumors have rapidly expanded. ${ }^{3-6}$ Thus, NGS is a powerful tool that can potentially provide an indication for targeted therapies and inform prognosis and family cancer risks. However, the interpretation of comprehensive NGS findings can be challenging and complex and can raise additional questions, even as it provides answers. This report presents several case studies illustrating important considerations in NGS testing in the solid tumor oncology setting.

\section{Interference of Clonal Hematopoiesis in Somatic Sequencing}

The first case involved an 80-year-old man with metastatic castration-resistant prostate cancer (mCRPC) whose disease was previously treated with abiraterone acetate and progressed on enzalutamide. He underwent biopsy of a bone metastasis in November 2018 with the hope of identifying potentially actionable mutations for additional treatment options. FoundationOne CDx (Foundation Medicine) testing of biopsy material revealed a well-studied $A T M$ c.9022C $>$ T (p.R3008C) pathogenic mutation $^{7}$ at $10 \%$ allelic fraction, but the sample was insufficient to assess microsatellite instability (MSI) or tumor mutational burden. The patient was seen in the clinic to discuss germline genetic testing and, as a result of the reported ATM mutation, consider targeted therapy clinical trial options, including several involving PARP inhibitors. Given the patient's interest in immune checkpoint inhibitors, a targeted gene NGS panel (UW-OncoPlex) validated for circulating tumor DNA (ctDNA) was sent to assess for MSI and tumor mutational burden in September 2019. The sample had a positive test result for 
2 presumed somatic mutations in $A T M$, c.9022C $>\mathrm{T}$ (p.R3008C) at $12 \%$ variant fraction and c.9021A $>$ T (p.E3007D) at 5\% variant fraction, and these were noted in trans (on different alleles). A peripheral blood DNA study shared the same ATM c.9022C $>$ T (p.R3008C) mutation reported in the prostate cancer metastatic bone biopsy, but the ATM c.9021A > T (p.E3007D) mutation had not previously been reported. It was discovered that for ctDNA testing, whole blood had inadvertently been drawn instead of plasma, the result of which was that lymphocytes constituted most of the DNA and would be expected to overwhelm the much smaller proportion of tumor-specific ctDNA. To clarify findings, plasma ctDNA (in which circulating cells are removed by centrifugation) was drawn. The same 2 mutations in ATM were identified: c.9022C $>$ T (p.R3008C) at $16 \%$ variant fraction and c.9021A $>$ T (p.E3007D) at 5\% variant fraction. The plasma cell-free DNA was compared with the reported results from the bone metastasis biopsy and whole blood. Although the first ATM c.9022C $>$ T (p.R3008C) mutation was identified in all 3 samples, the variant allelic fraction of the tumor-specific somatic variant should be higher in the plasma sample than in the whole blood sample and highest in the metastatic bone biopsy (Figure 1). However, this was not observed. Careful review of the variant allelic fractions from the 3 studies together showed that they were consistent with a clonal hematopoietic $(\mathrm{CH})$ process being the likely source of the $2 A T M$ clones and, importantly, unrelated to the prostate cancer.

Both ctDNA and metastatic biopsies can potentially be contaminated by $\mathrm{CH}$ and may be detected as minor populations in clinical NGS assays. Increasing evidence suggests that $\mathrm{CH}$ is a source of interference and should be routinely considered during interpretation of solid tumor NGS studies. ${ }^{8-14}$ A prospective study in which 124 patients with metastatic cancer underwent paired NGS of peripheral blood and tumor DNA with high-intensity sequencing (508 genes, $2 \mathrm{Mb},>60,000 \times$ raw depth) reported that $53 \%(918 / 1,727)$ of mutations in ctDNA samples were attributable to $\mathrm{CH} .{ }^{14}$ A large retrospective analysis of samples from patients with solid tumors reported that in the absence of matched blood control subjects, $5 \%(912 / 17,469)$ of patients would have had at least one $\mathrm{CH}$-associated mutation identified from ctDNA erroneously attributed to the solid tumor. ${ }^{8}$ The most frequently altered genes in $\mathrm{CH}$ were $D N M T 3 A$, TET2, PPM1D, ASXL1, TP53, SF3B1, SRF2, CHEK2, ATM, and $K M T 2 D .{ }^{8}$ In another study, $A T M$ mutations were deemed of likely $\mathrm{CH}$ origin in $20.9 \%$ (162 of 775 ) of individuals undergoing hereditary pan-cancer panel testing. ${ }^{9}$ Distinguishing between $\mathrm{CH}$ and cancer-associated mutations is of great clinical importance in both metastatic biopsies and ctDNA assays.
Different tumor specimen types can be used for NGS analysis, each with advantages and disadvantages (Table 1). This case illustrates how assessment of multiple samples, combined with expert interpretation and molecular tumor board review, was critical and allowed the patient to avoid a treatment targeting ATM mutations, which may have been ineffective due to interpretive analytic error rather than a bona fide tumor-specific driver mutation.

\section{Somatic Versus Germline}

The second case involved a 76-year-old man with highrisk localized prostate cancer (cT2b; Gleason score $4+$ $4=8$; prostate-specific antigen [PSA] level, $9.1 \mathrm{ng} / \mathrm{mL}$ ). This patient's family history was notable for an older sister diagnosed with breast cancer at age 73 years and his mother diagnosed with mesothelioma at age 80 years. He had no other family history of cancer, including among another sister and 2 daughters. The patient was part of a research study in which tumor DNA was evaluated using a comprehensive targeted gene NGS panel (UW-OncoPlex). The panel results revealed biallelic BRCA2 mutation: BRCA2 c.9196C > T (p.Q3066×) at $41 \%$ variant allelic fraction and BRCA2 c.7914del (p.P2639Lfs*9) at $29 \%$ variant allelic fraction. The discovery of $B R C A 2$ alterations, particularly at higher variant allelic fraction, prompted genetic counseling referral. Dedicated germline genetic testing confirmed that the BRCA2 c.9196C $>\mathrm{T}$ was a heterozygous germline variant and led to cascade genetic testing of his family. His older sister with breast cancer and one of his 2 daughters were found to carry the same germline BRCA2 c.9196C $>$ T mutation. These findings changed management in his sister's breast cancer treatment, his daughter's cancer surveillance, and finally the patient's own surveillance for other cancers. He completed definitive radiation therapy with androgen deprivation therapy (ADT) and currently has no signs of recurrence.

Both germline and somatic genetic testing may inform treatment decisions and prognosis for patients with cancer. Identifying potential germline mutations from tumor NGS can be clinically important. Reporting of suspected germline mutations varies across commercially available NGS platforms, with some laboratories reporting findings that may potentially be germline, other laboratories reporting both tumor and germline findings in aggregate and not calling attention to results that could be germline, and still other laboratories with bioinformatics set up to filter out germline data, thus removing the ability to identify germline variants. Clues that suggest potential germline origin of variants are relatively high variant allelic fraction (eg, $\sim 40 \%$ ), previous studies of variants in germline capacity (eg, ClinVar), and strong family history of cancer. 


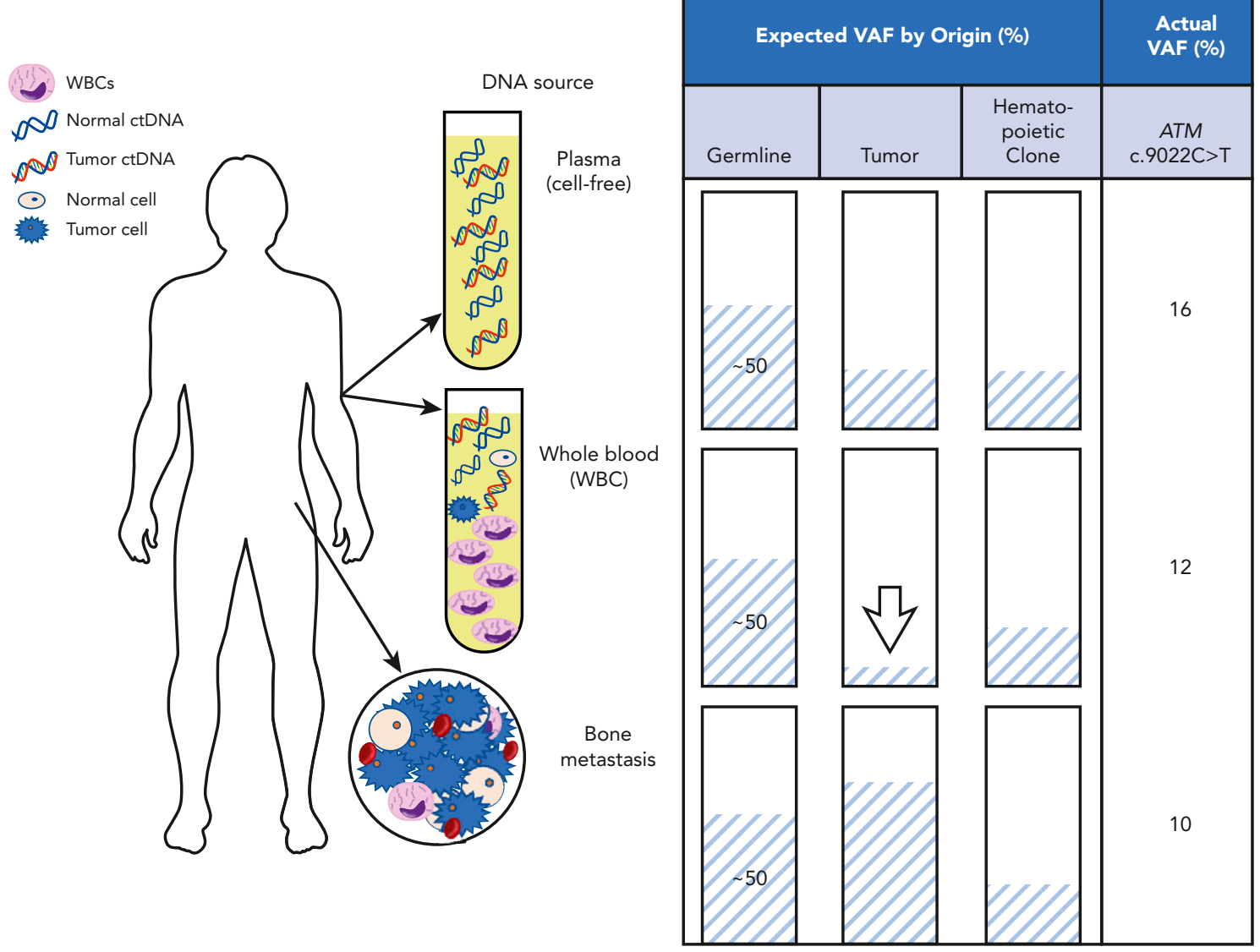

Figure 1. Conceptual model of VAF percentage in whole blood, plasma, and bone biopsy (with tumor purity $>80 \%$ ). Germline variants would be expected to have the same VAF in whole blood, plasma, and bone biopsy. Somatic tumor variants are expected to have significantly lower VAF in whole blood than in plasma because all blood cells are contributing to the denominator in whole blood samples and cells are removed in the plasma sample. Hematopoietic variants are expected to have the same VAF in whole blood and plasma.

Abbreviations: ctDNA, circulating tumor DNA; VAF, variant allelic fraction.

It should be noted that because of these differences in reporting across assays, the absence of germline variants/mutations on somatic testing is insufficient to make germline conclusions. Genetic counseling and dedicated germline testing are recommended if tumor NGS identifies a pathogenic variant in a gene associated with a cancer predisposition gene, especially if observed at high variant allelic fraction or there is other suspicion based on personal or family history. A special note of caution regarding TP53, which is a gene very commonly mutated in both $\mathrm{CH}$ and tumors and is rarely representative of the germline Li-Fraumeni syndrome: routine germline testing is not necessary for most patients with somatic TP53 mutations and should be reserved only for patients

\section{Table 1. Comparison of DNA Sources for Somatic Next-Generation Sequencing}

\begin{tabular}{|c|c|c|}
\hline Sample & Limitations & Advantages \\
\hline \multirow[t]{2}{*}{ Plasma ctDNA } & Sensitivity depends on context (correlates with tumor burden) & Minimally invasive \\
\hline & Specificity is uncertain (may reflect noncancer processes; eg, $\mathrm{CH}$ ) & May reflect composite of multiple progressing clones \\
\hline Primary tumor biopsy & $\begin{array}{l}\text { May not reflect changes arising later in oncogenesis (truncal/ } \\
\text { driver events may be detectable) }\end{array}$ & If available, no additional invasive procedure is required \\
\hline \multirow[t]{2}{*}{ Metastasis biopsy } & Invasive procedure required & May reflect dominant or progressing clonal population \\
\hline & May not reflect tumor heterogeneity & \\
\hline
\end{tabular}

Next-generation sequencing for CTCs is not clinically used. CTC enumeration is FDA-approved as a prognostic tool in metastatic breast, colorectal, and prostate cancer. CTCs are also being investigated for single-cell analysis, RNA sequencing, and prediction of response/recurrence.

Abbreviations: $\mathrm{CH}$, clonal hematopoiesis; $\mathrm{CTC}$, circulating tumor cell; ctDNA, circulating tumor DNA. 
with suspicious personal/family history and with genetic counseling involvement.

In this patient's case, expertise in tumor-germline NGS interpretation allowed recognition that the results were concerning for a possible germline $B R C A 2$ variant. This led to a referral to genetic counseling, confirmatory germline testing, and cascade family testing that identified the mutation in his sister and daughter and ultimately provided valuable information for both the patient and his family. This case also illustrates that men with prostate cancer can be the first in the family to be identified as carrying germline mutations such as BRCA2 and that cascade testing of relatives can be clinically important.

\section{Reclassification of Variants of Unknown Significance}

\section{Reclassification to Likely Benign}

The third case involved a 48-year-old woman diagnosed with breast cancer. She underwent germline testing and was found to have c.2658G $>$ T (p.Q866H) variant of unknown significance (VUS) in APC. On genetic testing, her 75-year-old mother was found to have the same VUS in APC. After learning about the APC VUS, the mother had an age-appropriate colonoscopy-her first colonoscopy-which did not identify colon polyps, arguing that the $A P C$ variant was unlikely to cause clinically actionable risk of polyposis. APC phenotype is highly penetrant, so a 75-year-old carrier without a clinical phenotype provided evidence that $A P C$ c. $2658 \mathrm{G}>\mathrm{T}$ (p.Q866H) should be reclassified as likely benign. It is important to note that discordant phenotypes alone are typically insufficient to downgrade VUS, but they may provide stronger evidence in cases of highly penetrant genes.

With the increasing use of multigene panel germline testing, we anticipate that more variants will be reported as VUS, which are challenging for patients to understand and for providers to resist overinterpreting within a clinical context. The latest information on variant relationships to phenotypes with supporting evidence can be found in freely accessible online databases: COSMIC (Catalogue of Somatic Mutations in Cancer) for somatic variants (cancer.sanger.ac.uk/cosmic) and ClinVar for germline variants (www.ncbi.nlm.nih.gov/clinvar/). ClinVar provides current information on variant pathogenicity classification.

VUS reclassification occurs via collaborative and labor-intensive work and most commonly results in VUS reclassification to likely benign/benign. ${ }^{15,16}$ The Find My Variant Study reported that $38(61 \%)$ of 63 variants were reclassified, with 32 (84\%) reclassified as benign/likely benign and $6(16 \%)$ as pathogenic/likely pathogenic. ${ }^{17}$
Through genetics professionals, patients with VUS can be encouraged to seek studies to help reclassify VUS, aid clinical management, and improve understanding of genotype-phenotype correlations. Studies can be found at ClinicalTrials.gov, such as the PROMPT study (ClinicalTrials.gov identifier: NCT02665195). Participating in clinical trials to reclassify VUS may be particularly important for patients with a strong family history of cancer, such as in the next case.

\section{Reclassification to Likely Pathogenic}

The fourth case involves a 50-year-old African American man diagnosed with T3aN1M0, Gleason $4+4$ prostate cancer. His sister had been diagnosed with breast cancer at age 40 years; his father and paternal uncle with prostate cancer in their 50s and 60s, respectively; his paternal first cousin with breast cancer at age 55 years; and his paternal grandmother with ovarian cancer (age unknown). Based on his personal and family history of cancer, he underwent panel NGS germline testing with tumor control. He was found to carry a germline $B R C A 2$ VUS, specifically BRCA2 c.8188G >C (p.A2730P).

The patient and his family were invited to participate in the Find My Variant research study to help reclassify the VUS. ${ }^{17}$ His sister with breast cancer had previously undergone germline testing and carried the same BRCA2 VUS. Segregation studies and familial cosegregation analysis were performed through the research study, and his paternal first cousin was found to also carry the variant. This indicated that the patient's father, who was diagnosed with prostate cancer, and a paternal uncle not known to have cancer were obligate carriers of the BRCA2 VUS. Integration of available data gave a $99 \%$ probability of BRCA2 variant pathogenicity. BRCA2 p.A2730P VUS was thus reclassified as likely pathogenic. Although the patient's family history was suggestive of a familial cancer syndrome and would prompt intensified cancer surveillance in family members, reclassification of $B R C A 2$ VUS expanded treatment options for the patient and his sister. This specific BRCA2 variant is more commonly observed in African American individuals, so the variant reclassification will help other families. Although VUS reclassification to likely pathogenic is rare, when there is a strong family history of cancer, participation in research studies such as in this case can help define less commonly seen variants. Concerted efforts to improve diversity of representation of available genetic databases will also help VUS reclassification.

\section{Molecular Markers for Response to Therapy}

The fifth case involved an 83-year-old man with mCRPC who initially presented with a PSA level of $317 \mathrm{ng} / \mathrm{mL}$, lymphadenopathy, and a bladder mass, which was biopsied to reveal Gleason score $5+4$ prostate adenocarcinoma. 
He initiated ADT and underwent tumor sequencing that showed MSI and hypermutation associated with biallelic $M S H 2$ mutation (exons 1-6 + EPCAM deletion). The result of germline testing for MSH2/EPCAM was negative. His prostate cancer developed resistance to ADT within 2 months and was refractory to both abiraterone acetate and enzalutamide. He received pembrolizumab as third-line treatment of mCRPC due to tumor MSI/hypermutation, but he did not achieve a clinical response to pembrolizumab.

The FDA approved pembrolizumab as the first tumor-agnostic therapy for patients with advanced solid tumor with deficient mismatch repair (dMMR) or $\mathrm{MSI}^{18}$ but did not specify a companion diagnostic or method used to determine dMMR/MSI. dMMR/MSI can be identified by immunohistochemistry, PCR, or NGS. In a retrospective series of 93 patients with colorectal cancer, $10 \%(n=9)$ were found to have a misdiagnosis of dMMR/ MSI status by local assessment, ${ }^{19}$ highlighting the need for standardization of methods to determine dMMR/ MSI. Possible discordance of results based on sample source and timing of the biopsy has been reported, suggesting that MSI/dMMR may develop later in the disease and be present in a metastatic tumor but not in the primary tumor. ${ }^{20}$

As this case illustrates, not all MSI/dMMR tumors respond to immune checkpoint inhibitors. This is corroborated by other studies, which in aggregate suggest a response rate to checkpoint blockade of approximately $50 \%$ among patients with MSI/dMMR. ${ }^{20,21}$ Further work is needed and is ongoing regarding as-yetundetermined factors that affect biologic consequences, primary resistance, and/or treatment response prediction. Models integrating multiple factors may perform better in predicting response to immune checkpoint inhibitors.

\section{Conclusions}

NGS is an emerging tool in clinical oncology. These case studies illustrate several key clinical and research considerations for interpretation of NGS results (Table 2):

- Mutations arising from contaminating hematopoietic clones may confound results from ctDNA and tumor/metastatic biopsies

- Tumor sequencing may raise suspicion for germline mutation and may call for confirmatory genetic counseling and testing

- Providers should be aware of potential follow-up opportunities for VUS reclassification, especially if there is a high pretest suspicion (eg, a strong family history of cancer)

- Shared expertise through molecular tumor boards may help in the accurate interpretation of complex NGS results after understanding the clinical context

The most extensive bioinformatics without clinical correlation and expert interpretation may lead to faulty conclusions and hamper clinical benefit. Recognition of the importance of these factors is reflected by emerging forums, such as the now-annual ASCO seminar, Genetics and Genomics for the Practicing Physician, first offered in 2018. Molecular tumor boards provide the opportunity for multidisciplinary discussion of patient medical history, family history, and NGS results to guide patientspecific treatment decisions, and should include faculty with expertise in genetics, laboratory medicine, pathology, and oncology. Commercial NGS platforms often provide access to their molecular tumor boards to discuss challenging cases. As NGS testing is increasingly deployed in clinical practice and data and informatics become denser and more complex, there is a need to create a network structure that will allow community

\section{Table 2. Strategies and Approaches to Address Selected Issues With NGS}

\begin{tabular}{|c|c|}
\hline Issue & Strategies and Approaches \\
\hline \multirow[t]{2}{*}{$\mathrm{CH}$ interference in ctDNA or tumor biopsy } & Request VAF \\
\hline & Interpret VAF in the context of available test results and clinical picture \\
\hline Tumor NGS is not universally reliable for germline variant identification & Dedicated germline testing is required for questions about inherited risk \\
\hline \multirow[t]{2}{*}{ Tumor NGS may raise suspicion of germline variant } & Counsel the patient on the possibility before somatic NGS \\
\hline & $\begin{array}{l}\text { If a germline variant is suspected, refer the patient to a genetic counselor for discussion } \\
\text { and dedicated germline testing }\end{array}$ \\
\hline \multirow[t]{4}{*}{ VUS reclassification } & $\begin{array}{l}\text { VUS should not be used for clinical management (rather, family history of cancer should } \\
\text { guide management) }\end{array}$ \\
\hline & Most VUS reclassifications are to benign or to likely benign \\
\hline & Suggest patient follow-up periodically for VUS reclassification \\
\hline & $\begin{array}{l}\text { Consider enrolling patient in studies for VUS reclassification, especially if a strong family } \\
\text { history of cancer is present }\end{array}$ \\
\hline
\end{tabular}

Abbreviations: $\mathrm{CH}$, clonal hematopoiesis; ctDNA, circulating tumor DNA; NGS, next-generation sequencing; VAF, variant allelic frequency; VUS, variant of unknown significance. 
oncologists to have access to academic and tertiary care center expert molecular tumor boards and keep pace with rapidly evolving knowledge and understanding.

\section{Acknowledgments}

We thank Dr. Stacy Cohen for helpful suggestions in facilitating case finding.

Submitted September 1, 2019; accepted for publication March 31, 2020.

Disclosures: Dr. Yu has disclosed that he receives consulting fees from Amgen, AstraZeneca, Bayer, Dendreon, Janssen, Merck, Pharmacyclics, and
Seattle Genetics, and grant/research support from Bayer, Dendreon, Merck, and Seattle Genetics. Dr. Cheng has disclosed that she receives grant/ research support from Clovis Oncology, Janssen, Medivation, Sanofi, and Astellas. The remaining authors have disclosed that they have not received any financial consideration from any person or organization to support the preparation, analysis, results, or discussion of this article.

Funding: This study was supported by generous funding from the Institute for Prostate Cancer Research, the Prostate Cancer Foundation, the National Cancer Institute (T32 CA009515, Training in Cancer Biology and Transplantation; CA097186, Pacific Northwest Prostate Cancer SPORE; Cancer Center Support Grant P30 CA015704), and the Congressional Designated Medical Research Program (PC131820).

Correspondence: Heather $\mathrm{H}$. Cheng, MD, PhD, Department of Medicine, University of Washington, 825 Eastlake Avenue East, Seattle, WA 98109. Email: hhcheng@uw.edu

\section{References}

1. Shaw AT, Riely GJ, Bang YJ, et al. Crizotinib in ROS1-rearranged advanced non-small-cell lung cancer (NSCLC): updated results, including overall survival, from PROFILE 1001. Ann Oncol 2019;30: 1121-1126.

2. Dummer R, Ascierto PA, Gogas HJ, et al. Overall survival in patients with BRAF-mutant melanoma receiving encorafenib plus binimetinib versus vemurafenib or encorafenib (COLUMBUS): a multicentre, open-label, randomised, phase 3 trial. Lancet Oncol 2018;19: 1315-1327.

3. Kindler $\mathrm{HL} T$, Hammel $\mathrm{P}$, Reni $\mathrm{M}$, et al. Olaparib as maintenance treatment following first-line platinum-based chemotherapy (PBC) in patients (pts) with a germline BRCA mutation and metastatic pancreatic cancer (mPC): phase III POLO trial [abstract]. J Clin Oncol 2019; 37(Suppl):Abstract LBA4

4. Domchek SM, Aghajanian C, Shapira-Frommer R, et al. Efficacy and safety of olaparib monotherapy in germline BRCA1/2 mutation carriers with advanced ovarian cancer and three or more lines of prior therapy. Gynecol Oncol 2016;140:199-203.

5. Armstrong AC, Clay V. Olaparib in germline-mutated metastatic breast cancer: implications of the OlympiAD trial. Future Oncol 2019;15: 2327-2335

6. Pritchard CC, Mateo J, Walsh MF, et al. Inherited DNA-repair gene mutations in men with metastatic prostate cancer. N Engl J Med 2016;375: 443-453.

7. Angèle $S$, Laugé $A$, Fernet $M$, et al. Phenotypic cellular characterization of an ataxia telangiectasia patient carrying a causal homozygous missense mutation. Hum Mutat 2003;21:169-170.

8. Ptashkin RN, Mandelker DL, Coombs CC, et al. Prevalence of clonal hematopoiesis mutations in tumor-only clinical genomic profiling of solid tumors. JAMA Oncol 2018;4:1589-1593.

9. Slavin TP, Coffee B, Bernhisel R, et al. Prevalence and characteristics of likely-somatic variants in cancer susceptibility genes among individuals who had hereditary pan-cancer panel testing. Cancer Genet 2019; 235-236:31-38

10. Hu Y, Ulrich BC, Supplee J, et al. False-positive plasma genotyping due to clonal hematopoiesis. Clin Cancer Res 2018;24:4437-4443.
11. Coombs CC, Zehir A, Devlin SM, et al. Therapy-related clonal hematopoiesis in patients with non-hematologic cancers is common and associated with adverse clinical outcomes. Cell Stem Cell 2017;21: 374-382.e4.

12. Coombs CC, Gillis NK, Tan X, et al. Identification of clonal hematopoiesis mutations in solid tumor patients undergoing unpaired next-generation sequencing assays. Clin Cancer Res 2018;24:5918-5924

13. Xie M, Lu C, Wang J, et al. Age-related mutations associated with clonal hematopoietic expansion and malignancies. Nat Med 2014;20: 1472-1478

14. Razavi $\mathrm{P}$, Li BT, Brown DN, et al. High-intensity sequencing reveals the sources of plasma circulating cell-free DNA variants. Nat Med 2019;25 1928-1937.

15. Mersch J, Brown N, Pirzadeh-Miller S, et al. Prevalence of variant reclassification following hereditary cancer genetic testing. JAMA 2018 320:1266-1274.

16. Slavin TP, Manjarrez S, Pritchard CC, et al. The effects of genomic germline variant reclassification on clinical cancer care. Oncotarget 2019 10:417-423. https://doi.org/10.18632/oncotarget.26501

17. Tsai GJ, Rañola JMO, Smith C, et al. Outcomes of 92 patient-driven family studies for reclassification of variants of uncertain significance. Genet Med 2019:21:1435-1442.

18. U.S Food and Drug Administration. FDA grants accelerated approval to pembrolizumab for first tissue/site agnostic indication. Accessed July 10 2020. Available at: https://www.fda.gov/drugs/resources-informationapproved-drugs/fda-grants-accelerated-approval-pembrolizumab-firsttissuesite-agnostic-indication

19. Cohen $R$, Hain $E$, Buhard $O$, et al. Association of primary resistance to immune checkpoint inhibitors in metastatic colorectal cancer with misdiagnosis of microsatellite instability or mismatch repair deficiency status. JAMA Oncol 2019;5:551-555.

20. Abida W, Cheng ML, Armenia J, et al. Analysis of the prevalence of microsatellite instability in prostate cancer and response to immune checkpoint blockade. JAMA Oncol 2019;5:471-478.

21. Le DT, Durham JN, Smith KN, et al. Mismatch repair deficiency predicts response of solid tumors to PD-1 blockade. Science 2017;357:409-413. 